\title{
Globe
}

Revue internationale d'études québécoises

\section{Entre plaisir et danger. Exploration de la sexualité au Québec à travers ses images et ses représentations}

\section{Julie Lavigne}

Volume 12, numéro 2, 2009

Images et représentations de la sexualité au Québec

URI : https://id.erudit.org/iderudit/1000704ar

DOI : https://doi.org/10.7202/1000704ar

Aller au sommaire du numéro

Éditeur(s)

Globe, Revue internationale d'études québécoises

ISSN

1481-5869 (imprimé)

1923-8231 (numérique)

Découvrir la revue

Citer ce document

Lavigne, J. (2009). Entre plaisir et danger. Exploration de la sexualité au Québec à travers ses images et ses représentations. Globe, 12(2), 11-21.

https://doi.org/10.7202/1000704ar d'utilisation que vous pouvez consulter en ligne.

https://apropos.erudit.org/fr/usagers/politique-dutilisation/ 


\title{
INTRODUCTION.
}

\author{
ENTRE PLAISIR
}

ET DANGER.

EXPLORATION DE LA SEXUALITÉ

AU QUÉBEC

\author{
À TRAVERS SES IMAGES \\ ET SES REPRÉSENTATIONS
}

JULIE LAVIGNE
Universite du Quebec à Moncreal
++++++++++++++++++++++++++++

Pourquoi s'intéresser à la représentation de la sexualité au Québec et particulièrement maintenant ? En fait, le présent numéro est né de plusieurs interrogations provenant au départ de la discipline de l'histoire de l'art féministe. Bien que la sexualité ait toujours fait partie intégrante du développement des arts, avec ses soubresauts de censure et d'exploration érotique, il appert qu'une mutation s'opère dans l'art contemporain dès les années 1970, mais surtout vers les années 1990 .

Le début des années 1970 est culturellement associé à trois mouvements sociaux précis: la libération sexuelle, l'essor d'un féminisme radical s'intéressant de plus en plus à la sexualité et le début du phénomène cinématographique hardcore que plusieurs théoriciens relient à la sortie du film de Gérard Damiano, Deep Throat, en $1972^{1}$. Cette effervescence culturelle marquera profondément la pratique de l'art féministe, notamment chez

$$
\div+\div
$$

1. Jacques Zimmer, Le Cinéma érotique, Paris, J'ai lu, 1988, p. 96-97; Linda WILlLaMS, Hard Core: Power, Pleasure and "the Frenzy of the Visible", Berkeley, University of California Press, 1989, p. 25 et 98. 
Judy Chicago, Carolee Schneemann, Valie Export, Lynda Benglis, pour ne nommer que quelques noms. Au Québec, c'est en 1976 que Francine Larivée exposera son ambitieux projet $L a$ chambre nuptiale au complexe Desjardins, une installation monumentale traitant de sexualité, d'oppression de la femme dans le couple et dans la famille ${ }^{2}$.

L'art féministe est marqué, dans les années 1990, par un retour au corps, un corps parfois très sexualisé. Dans leur ouvrage de référence Art and Feminism, Peggy Phelan et Helena Reckitt qualifient les années 1990 comme suit:

Au cours des années 1990 , le corps sexué est de nouveau considéré comme source d'inspiration artistique - non plus comme un objet fétichisé par le regard de l'homme, mais comme un élément à explorer dans toute sa diversité. Le corps des années 1990 est animé par la reconnaissance d'une perversité polymorphe et par le futuriste cyborg ${ }^{3}$.

Effectivement, depuis les années 1990, la mise en scène du corps et de la sexualité refait surface dans l'art féministe avec des artistes comme Annie Sprinkle, Pipilotti Rist, Marlene Dumas, Helen Chadwick, Zoe Leonard ou encore Jenny Saville. Ce retour au corps sexualisé, et dans certains cas de manière explicite, concorde aussi avec l'émergence d'une troisième vague féministe. Bien que le terme "vague» soit loin de faire consensus dans les études féministes, il n'en demeure pas moins que, dans les années 1990, un bassin de théoriciennes abordera la sexualité d'une manière plus positive, notamment en faisant preuve d'ouverture sur les questions épineuses du travail du sexe, de la pornographie et de la sexualité adolescente ou marginale. D'ailleurs, le texte de Louise Toupin présenté dans ce numéro cristallise bien la rension entre ce féminisme plus ouvert aux questions sexuelles et un féminisme radical plus traditionnel. Les théoriciennes étiquetteront ces nouvelles féministes de "prosexe $\mathrm{e}^{4}$ ", de libertaires ${ }^{5}$ ou encore

$$
+4
$$

2. Voir sur cette cuvre, entre autres, le texte "Dissidence et différence: aspects de l'art des femmes" de Rose Marie ARBour, dans Marie-Charlotte DE KONNING et Pierre LANDRY, Déclics. Art et société. Le Québec des années 1960 et 1970, Montréal, Éditions Fides, 1999, p. 114-145.

3. "The 1990s renewed interest in the gendered body as an artistic source - no longer as the fetishized object of the male gaze but as something to be explored in all of its diversity. The 1990s body was infused with consciousness of polymorphous perversity and cyborg futures" (Peggy PHELAN et Helena RECKITT, Ant and Feminism, Londres et New York, Phaidon Press, 2001, p. 156).

4. Gayle S. Rubin, "Penser le sexe", Gayle S. RubiN et Judith Butler, Marché au sexe, Paris, EPEL, 2001 [1984], p. 120.

5. Steven SEIDMAN, Embattled Eros. Sexual Politics and Ethics in Contemporary America, Londres et New York, Routledge, 1992, p. 145-205. 
de féministes de la troisième vague ${ }^{6}$. Par ailleurs, les années 1990 verront la pornographie hardcore entrer dans l'ère numérique et prendre une ampleur considérable. Un tel phénomène suscitera l'intérêt des artistes et modifiera nécessairement l'horizon d'artente des images à caractère sexuel. Des artistes comme Robert Mapplethorpe, Jeff Koons, Annie Sprinkle ou Natacha Merritt utiliseront la pornographie en art au point de rendre caduque toute distinction entre l'art et la pornographie. Outre ces exemples extrêmes, la sexualité et sa représentation plus ou moins explicite sont à la source de nombreux projets en art contemporain en Occident, et ce, surtout depuis les deux dernières décennies.

Cependant, cette thématique ne semble pas avoir retenu beaucoup l'attention au Québec. Mis à part des œuvres éparses de quelques artistes comme Geneviève Cadieux, Jana Sterbak, David Altmejd, Patrick Bernatchez ou Cooke-Sasseville, peu de travaux en art visuel se consacrent vraiment à l'exploration de la sexualité et encore moins à la représentation d'une sexualité explicite ou d'images pornographiques. Pourtant, comme en témoignent les articles d'Évelyne Ledoux-Beaugrand, d'Isabelle Boisclair et de Martine Delvaux figurant dans ce numéro, le phénomène d'une représentation plus explicite de la sexualité existe en littérature. Est-ce la transposition de cette sexualité explicite en images qui pose problème au Québec? À en croire l'adaptation cinématographique édulcorée des romans de Marie-Sissi Labrèche analysée par Ledoux-Beaugrand, il semble que la menace de la censure demeure prégnante. Toutefois, à l'inverse du cinéma, les arts visuels ne font face à aucun mécanisme de censure externe; il appert ainsi que les artistes font preuve d'une plus grande autocensure lorsqu'il s'agit de mettre en scène une sexualité explicite.

En dehors du milieu des arts, la situation revêt un tout autre visage. En mai 2008, le Conseil du statut de la femme publiait un avis intitulé Le sexe dans les médias: obstacle aux rapports égalitaires ${ }^{7}$, un texte qui fait l'objet d'une analyse critique de Maria Nengeh Mensah dans la section "Perspective" de ce numéro. L'avis du Conseil sonnait alors l'alarme sur la présence massive de la sexualité dans les médias. Qui plus est, il s'agissait, selon le document, d'une sexualité inégalitaire, voire sexiste. Un an plus tôt,

$$
+++
$$

6. Maria Nengeh MENSAH, Dialogues sur la troisième vague féministe, Montréal, Les éditions du remueménage, 2005, p. 16.

7. Ginetre Plamondon, Annie Desaulniers ex Nathalie Roy, Le sexe dans les médias: obstacles aux rapports égalitaires, Québec, Conseil du stacut de la fermme, 2008. 
un documentaire, Sexy Inc. Nos enfants sous influence ${ }^{8}$, qui a également fait grand bruit, abordait l'hypersexualisation des jeunes, l'omniprésence d'une sexualité explicite dans les médias et les conséquences néfastes de cette prétendue surexposition sur les jeunes. $\grave{A}$ en croire ces deux recherches ainsi que de nombreux autres travaux ${ }^{9}$, il y aurait effectivement trop de représentations de la sexualité dans les médias au Québec. L'article de Martin Blais et collaboratrices ainsi que celui d'Eve Paquette examinent en détail le discours médiatique et scientifique autour de l'hypersexualisation et de la relation des jeunes avec la pornographie sur Internet afin de remettre en question certains présupposés et de dénoncer une forme de panique morale.

Comment comprendre ceci, qui peut sembler paradoxal : d'une part, des cinéastes qui craignent la censure et des artistes qui s'autocensurent et, d'autre part, des scientifiques et des journalistes ${ }^{10}$ qui dénoncent avec vigueur le fait que les médias regorgent d'une sexualité de plus en plus explicite? Le sociologue de la sexualité, Michel Bozon, offre une piste des plus pertinentes pour comprendre l'écart entre deux phénomènes qui paraissent effectivement contradictoires. Selon Bozon, dans la société actuelle trois types parfois antinomiques de construction ou de compréhension de la sexualité ont cours. Ces constructions, que Bozon nomme des orientations intimes de soi, "constituent de véritables cadres mentaux, qui délimitent l'exercice de la sexualité, définissent le sens qui lui est donné et indiquent le rôle que la sexualité joue dans la construction de soi ${ }^{11}$ ". Il s'agit en quelque sorte de barèmes régissant les représentations et les interactions sociales ainsi que les représentations de soi. À ses yeux, il existerait donc trois genres d'orientation intime de soi : le réseau sexuel, le désir individuel et la sexualité conjugale. En résumé, l'orientation intime de soi de type réseau sexuel présente des individus ayant une sociabilité fortement teintée par la vie sexuelle et une sexualité extravertie. Le modèle d'orientation du désir individuel est en quelque sorte le modèle inverse puisque l'expression de la

$$
+*
$$

8. Sylvie BISSONNETTE, Sexy Inc. Nos enfants sous influence, Québec, Office national du film, 2007, $35 \mathrm{~min}$.

9. Voir notamment les recherches de Richard Poulin, Pierrette Bouchard, Natasha Bouchard et Isabelle Boily ainsi que celles de Francine Duquet et d'Anne Quéniart.

10. Outre le documentaire Sexy Inc., au moins trois autres documentaires metcent de l'avant un discours similaire: Pierre COTE, Trop jeunes pour être sexy, Société Radio-Canada, Enjeux, 14 janvier 2003; TELEQUÉBEC, Porno et ado: un mélange explosif?, Kilomètre Zéro, 27 octobre 2008; Michel VINCENT, Adoporno.com, Société Radio-Canada, Enjeux, 28 octobre 2003. Plusieurs numéros spéciaux de revue d'intérêt général ou de journaux ont également diffusé ce type d'idées: La Gazette des femmes, vol. 27, n² 2, septembre-octobre 2005; un cahier spécial du Devoir, 16-17 avril 2005; le numéro thématique "Sexe, école et pornon de $A$ Babord. Revue sociale es polttique, $n^{\circ} 20$, été 2007.

11. Michel BOzON, Sociologie de la sexualite, Paris, Armand Colin, 2005, p. 108. 
sexualité y est moins extravertie, plus orientée vers le désir et le plaisir de l'individu. L'orientation intime de soi axée sur la sexualité conjugale privilégie, quant à elle, la sexualité comme moteur de la vie conjugale; elle ne s'exprime qu'à l'intérieur du couple. Or, pour Bozon, ces trois orientations intimes de soi donnent lieu à trois types très différents de conceptualisation sociale de la sexualité et de ses représentations. En effet:

D'un côté, la sexualité contemporaine est dénoncée car elle entraînerait le nomadisme sexuel des individus, la tyrannie du plaisir et du désir, la permissivité des mœurs, voire la "dévirilisation" des hommes. Ces critiques s'appuient sur une perspective conjugale stricte pour condamner radicalement, comme manifestation typique d'une perte de valeurs contemporaine, les comportements et les attitudes que nous avons rattachés à la perspective du réseau et à celle du désir. Mais cette grille de lecture peut fort bien être renversée et les transformations contemporaines être lues positivement comme une "révolution sexuelle", consacrant le droit au plaisir, l'égalité sexuelle entre femmes et hommes, dans le cadre d'un accès généralisé à la contraception; selon cette interprétation quelque peu messianique, qui peut être rattachée à l'orientation du désir individuel ou à celle de la sociabilité sexuelle, c'est au contraire la période précédente qui doit être considérée comme un âge de répression, d'hypocrisie et de tabou ${ }^{12}$.

Avec un tel écart entre les diverses conceptualisations, il n'est pas étonnant que les discussions qui tournent autour de la sexualité soient aussi enflammées. Des discussions ont effectivement lieu en France entre autres autour de la pornographie, de l'industrie du sexe, de l'étatisation de l'éducation sexuelle ou de la relation des jeunes avec la sexualité ${ }^{13}$. Ces débats prennent une tournure encore plus dramatique lorsqu'il s'agit des relations des jeunes avec la sexualité et ses représentations pornographiques. Comme le soulignait déjà Gayle Rubin en 1984, "depuis plus d'un siècle, aucune stratégie pour susciter l'hystérie sexuelle n'a fonctionné aussi bien que l'appel à protéger les enfants ${ }^{14} n$.

$$
+4
$$

12. Michel BOzON, "Orientations intimes et constructions de soi. Pluralicé et divergences dans les expressions de la sexualité", Sociétés Contemporaines, $n^{\text {os }}$ 41-42, 2001, p. 29.

13. Sur le sujet, on peut lire: Christian AUTHIER, Le nouvel ordre sexueh Paris, Bartillat, 2002; Xavier DELEU, Le consensus pormographique, Paris, Mango, 2002; Marcela LACUB et Patrice MANGLIER, Antimanuel d'éducation sexuelle, Paris, Editions Bréal, 2005; Michela MARZANO, La pormographie ou l'épuisement du désir, Paris, Buchet-Chastel, 2003; Ruwen OGIEN, Penser la pornographie, Paris, Presses Universitaires de France, 2003; La panique morale, Paris, Éditions Grasset, 2004.

14. Gayle S. RUBIN, "Penser le sexe", Gayle S. RUBIN et Judith BUTLER, Marché au sexe, op. cit., p. 72. 
Bien que la discussion entre les deux camps - d'une part, les tenants d'une conception conjugale de la sexualité et, d'autre part, les tenants d'une vision plus individualiste ou axée sur la "sociabilité sexuelle" - relève souvent d'un dialogue de sourds, il n'en demeure pas moins que des débats ont lieu non seulement en France, mais aussi aux États-Unis ${ }^{15}$, et que les deux visions discordantes entourant la sexualité, ses images et les jeunes ont droit de cité. Malheureusement, ce n'est pas vraiment le cas au Québec, du moins en ce qui concerne la sexualité dans les médias et la notion d'hypersexualisation. Depuis l'émergence de ce concept et l'insistance médiatique sur les dangers de la pornographie sur Internet, il n'y a eu que très peu de contre-discours critiques pertinents sur ces sujets. Or, dans une société aussi pluraliste que la nôtre - que les sociologues de la sexualité John Gagnon et William Simon qualifiaient de société postparadigmatique (donc non traditionnelle, ou moderne) -, il est impératif d'entendre divers points de vue sur des phénomènes aussi sensibles. Le présent numéro entreprend donc de délaisser la voie de la morale pour explorer les images et les représentations sexuelles dans une perspective d'éthique minimale ${ }^{16}$, ou des éthiques sexuelles contemporaines ${ }^{17}$, comme le suggèrent respectivement Ruwen Ogien et Valérie Daoust.

\section{PRÉSENTATION DU NUMÉRO}

Le texte de Martin Blais, Sarah Raymond, Hélène Manseau et Joanne Otis s'inscrit parfaitement dans cette voie et aborde l'analyse du concept d'hypersexualisation sous un nouvel angle. En effet, leur article décrit, dans un premier temps, le phénomène médiatique et universitaire que l'on

$$
++
$$

15. Par exemple, le féminisme américain est divisé depuis les années 1980 par ce que les théoriciens et théoriciennes appellent la Sex War. Ce débat autour de la permissivité, de l'éducation sexuelle, de la sexualité des jeunes, fait également couler beaucoup d'encre aux États-Unis. Sur la Sex War, voir Gayle S. RUBIN ec Judith BUTLER, Marché au sexe, op. cit.; Steven SEIDMAN, Embattled Eros, op. cit. et Maria Nengeh MENSAH, Dialogues sur la troisième vague fémintste, op. cit. Pour un débat plus large, voir notamment Jeffrey WEEKS, Sexuality and its Discontents: Meanings, Myths and Modern Sexualities, Londres, Roucledge, 1985; Alan SOBLE et Nicholas POWER (dir.), The Philosophy of Sex. Contemporary Readings, Lanham, Rowman \& Littlefield Publisher, 2008.

16. Le concept d'éthique minimale développé par Ruwen OGIEN apparaît pour la première fois dans l'essai d'éthique appliquée à la pornographie Penser la pornographie (op. cit.). En réaction à des philosophes liberaux se positionnant contre la pornographie, Ogien propose d'aborder le phénomène selon une approche répondant à trois principes fondamentaux: «1) La neutralicé à l'égard des conceptions substantielles du bien; 2) Le principe négatif d'éviter de causer des dommages à autrui ; 3) Le principe positif qui nous demande d'accorder la même valeur aux voix ou aux intérêts de chacun" (p. 12). Ces trois principes permettraient selon Ogien d'évaluer moralement la pornographie tout en demeurant fidèle au principe libéral de neutralité vis-à-vis des conceptions du bien, particulièrement important dans le champ des pratiques sexuelles.

17. Voir Valérie DAOUST, De la sexualité en démocratie. L'individu libre et ses espaces identitaires, Paris, Presses Universitaires de France, 2005. 
nomme désormais hypersexualisation. Les auteurs entreprennent ensuite de vérifier, à l'aide des données empiriques disponibles, quatre affirmations précises qui soutiennent ce discours sur la sexualité des jeunes: 1) les jeunes font une entrée plus précoce dans la vie sexuelle active; 2 ) leurs activités sexuelles sont banalisées; 3 ) on assiste à " une perte de repères et au déclin des valeurs sociomorales sur la sexualité et le couple"; 4) les représentations sexualisées se font de plus en plus envahissantes au sein des médias. Selon les auteurs, seule la dernière affirmation est appuyée par les données empiriques, quoiqu'il faille la nuancer, alors que les trois autres affirmations sont littéralement invalidées: en fait, la majorité des jeunes ont une vie sexuelle et des valeurs sociomorales similaires à celles des générations précédentes.

Dans le même esprit, le texte d'Eve Paquette aborde le thème du numéro dans une perspective plus circonscrite, en s'intéressant au rapport des adolescents à l'usage sexuel d'Internet. Plutôt que d'en vérifier la validité empirique, l'auteure aborde la problématique de l'hypersexualisation par une analyse du discours médiatique. Plus précisément, elle se penche sur deux documentaires de l'émission télévisuelle Enjeux consacrés aux jeunes, à leur sexualité et à leur usage d'Internet ("Les jeunes à l'école du sexe" [2000] et "Adoporno.com" [2003]) et sur le discours médiatique sur le prosélytisme pédophile sur Internet. En prenant notamment appui sur les théories de Paul Ricœur sur l'imaginaire de la souillure, Paquette expose la tendance des discours médiatiques à entretenir à la fois l'image de l'adolescent innocent et celle du danger d'Internet, qui serait, lui, source de souillure de la sexualité des adolescents. Selon l'auteure, ce discours tient d'une panique morale plus que d'un réel danger social.

Le caractère malsain du couplage sexualité et Internet est au cœur du deuxième roman de Nelly Arcan, analysé par Isabelle Boisclair. La lecture attentive que présente Boisclair de Folle s'articule principalement autour de la primauté du désir masculin, de la négation du corps et de la subjectivité de la femme dans le contexte du recours à la cyberpornographie. Fidèle à l'esprit du roman d'Arcan, Boisclair repose sa lecture sur une conception féministe négative et pessimiste de la cyberpornographie, comme en témoigne le recours aux idées de Marzano et MacKinnon sur cette thématique. Par ailleurs, compte tenu du caractère autofictionnel de certains aspects du roman, entre autres par l'utilisation du pseudonyme "Nelly", l'analyse de Boisclair résonne de manière singulière, car Radio-Canada annonçait ce matin même (25 septembre 2009) le suicide de Nelly Arcan. Sans vouloir réinterpréter le roman à la lumière de cet événement biographique, on peut tout de même avancer que "la déshumanisation des femmes à laquelle mène 
la cyberpornographie" et la négation du corps que la narratrice subit et s'auto-inflige dans Folle revêtent un sens d'autant plus dramatique. Cependant, l'auteure prend soin de relever la résistance de la narratrice qui s'exprime certes par une intériorisation de la norme, mais une intériorisation explicite, consciente et assumée par la protagoniste. Dès lors, il ne peut s'agir que d'une narratrice consciente de la norme qu'elle endosse en partie puisqu'elle y résiste par une prise de parole explicite dans certains passages et par l'écriture même du roman.

$\mathrm{Au}$ Québec, lorsque l'on évoque la représentation explicite de la sexualité en littérature, deux noms surgissent immédiatement: Nelly Arcan et Marie-Sissi Labrèche. L'article d'Évelyne Ledoux-Beaugrand aborde l'œuvre écrite de Labrèche dans une perspective comparatiste qui vise à interpréter les écarts entre l'esthétique des romans et celle de leur adaptation cinématographique par Lyne Charlebois. Alors que l'interprétation du roman d'Arcan appelait une approche féministe dénonçant les effets de la pornographie, l'analyse des romans de Labrèche se fait sous l'angle libertaire de la pornographie. En effet, Ledoux-Beaugrand fait état de l'esthétique postpornographique des romans de Labrèche. Le concept de postpornographie a été élaboré par une théoricienne queer, Marie-Hélène Bourcier, et fait référence à la pratique pornographique, féministe et critique qui détourne les codes de la pornographie conventionnelle (sexualité explicite, vulgarité, excès visuel et gros plan sur les organes génitaux, par exemple). Or, selon Ledoux-Beaugrand, l'esthétique "postporno" et transgressive centrée sur une sexualité féminine excessive et constitutive de l'œuvre littéraire laisse place, dans son adaptation cinématographique, à une esthétique léchée et édulcorée qui évite la représentation explicite, sublimant ainsi la quête sexuelle en une quête amoureuse. Bien que la menace d'une censure, similaire à celle qu'a subie en France le film Baise-moi, aurait été réelle si l'adaptation cinématographique de Charlebois avait été plus fidèle à la lettre des romans de Labrèche, l'auteure interprète l'écart entre les romans et le film comme une censure désormais indirecte qui relèverait d'une crainte de la contamination de la société par une représentation explicite d'une sexualité atypique et, de surcroît, féminine.

Alors que Boisclair aborde l'excès de subjectivité et de corps (c'està-dire le corps dans toute sa matérialité, et non plus virtuel) de la femme par rapport à la norme cyberpornographique dans le roman d'Arcan et que Ledoux-Beaugrand traite de l'excès de sexualité subversive du personnage féminin dans les romans de Labrèche, le texte de Martine Delvaux s'intéresse à l'excès d'organicité des femmes à travers une étude de la figure de la 
poupée. À partir du concept de singularité quelconque de Giorgio Agamben, Delvaux propose de comprendre la figure de Barbie et surtout de la RealDoll-poupée en silicone hyperréaliste pour adulte - comme le réceptacle par excellence, car muet, des fantasmes masculins, une a-identité féminine niant toute subjectivité ou humanité aux femmes. En contreexemple de cette version plastifiée et parfaite d'une féminité muette, l'auteure présente les «filles sans nom " qui peuplent les poèmes de Josée Yvon. Même sans nom, les femmes d'Yvon représentent l'envers de la figure fétichisée de la poupée. En effet, Yvon met en scène des femmes bien réelles, meurtries, enragées et prêtes à exploser: cette exacte part irreprésentable des femmes que tente d'oblitérer Barbie ou sa version pour adulte.

Le texte de Louise Toupin se penche également sur une voix subversive des femmes, celle des travailleuses du sexe. Plus particulièrement, l'auteure s'intéresse à l'émergence du sujet politique de la prostituée, de la lutte pour les droits de ces travailleuses et des problèmes d'intégration de cette nouvelle "parole publique" dans le mouvement des femmes au Québec. Alors que des organismes comme Stella voient le jour afin d'aider les travailleuses du sexe à avoir les meilleures conditions de travail possible, et ce, dans une perspective féministe, le mouvement des femmes, largement influencé par le courant abolitionniste, peine à reconnaître la légitimité même de cette voix féministe hétérogène. Or, ce rejet, selon Toupin, n'est pas sans poser problème. Il s'agit fondamentalement d'un rejet de la subjectivité des travailleuses du sexe: le mouvement des femmes ne voit en elles que les victimes du système prostitutionnel, détournant même leurs discours et leurs revendications afin qu'ils correspondent au cadre conceptuel abolitionniste. Ce rejet témoigne d'un manque d'ouverture du mouvement des femmes à l'égard de l'hétérogénéité des réalités des femmes. Pour Toupin, la difficile intégration de la voix politique des travailleuses du sexe résulte de la persistance du "stigmate de putain", voire d'une re-stigmatisation de la mauvaise fille au nom même du féminisme.

Le texte de Jean-Philippe Warren examine le discours sur la sexualité dans la revue Parti pris, permettant ainsi de compléter le numéro par une étude qui se penche davantage sur la masculinité. L'intérêt du texte réside aussi dans le fait qu'il explore un corpus plus historique, les années 1960, et un sujet plus canonique en études québécoises, soit la Révolution tranquille et la revue Parti pris. La perspective d'analyse privilégiée par l'auteur est novatrice, car Warren s'intéresse à la sexualité et à ses métaphores, qui alimenteront les visées émancipatrices des collaborateurs de la revue. Plus concrètement, le sociologue se penche sur les articles afin d'en "dégager les 
représentations de la sexualité ". Il y est évidemment question de révolte envers le contrôle religieux et la persistance d'une morale janséniste, mais aussi des effets de la colonisation sur la sexualité et la masculinité. Dans une rhétorique machiste et hétérosexiste qui n'est pas sans rappeler le discours masculiniste contemporain, la décolonisation du peuple québécois (entendre ici des hommes du Québec) passait, pour les partipristes, par la révolution sexuelle, par une réaffirmation de la masculinité toute-puissante et par un rétablissement de la figure du Père au sens psychanalytique du terme. Tout en dénonçant le caractère misogyne et homophobe des propos partipristes, Warren s'efforce de comprendre, par le contexte politique et social, l'émergence de cette voix politique singulière.

Pour les chroniques «Perspective" et " Note critique», j'ai fait appel à deux chercheurs prolifiques et incontournables dans le champ de recherche que constitue la sexualité au Québec. La chronique "Perspective", sous la plume de Maria Nengeh Mensah, s'attaque à la teneur du dernier avis du Conseil du statut de la femme sur Le sexe dans les médias et au phénomène médiatique autour de l'hypersexualisation. Dans le même esprit que les textes de Blais et collaboratrices et de Paquette, Mensah dénonce ce qu'elle qualifie d' "hypermoralisme" dans le discours sur la relation entre la sexualité, les jeunes et les médias. S'appuyant sur les concepts de dispositif de la sexualité de Michel Foucault et de morale sexuelle substantialiste de Marcela Iacub, Mensah fait ressortir que l'avis du Conseil du statut de la femme, sous prétexte de promouvoir une sexualité égalitaire pour les jeunes, sème une panique morale ayant "pour tâche de prohiber les manifestations visibles de la sexualité, le sexe dans l'espace public». La "Note critique", quant à elle, aborde un thème qui constitue une part importante des études sur la sexualité, mais qu'aucun autre texte du dossier n'aborde de front: l'homosexualité au Québec. Joseph Josy Lévy et Catherine de Pierrepont font la recension de trois ouvrages qui s'intéressent à différents aspects de l'homosexualité au Québec. Le premier, celui de Patrice Corriveau, La répression des homosexuels au Québec et en France. Du bûcher à la mairie, retrace, dans une perspective comparatiste, l'évolution historique du statut sociopolitique des homosexuels ou de leur reconnaissance. Les deux autres ouvrages se concentrent sur une question plus précise, soit le mariage gai. La monographie éditée par Guy Ménard, Mariage homosexuel. Les termes du débat, regroupe 45 textes publiés ou reçus par Le Devoir et traitant du mariage gai, alors que le livre Mariage gai. Les coulisses d'une révolution sociale, de Sylvain Larocque, décrit de manière journalistique les luttes politiques, juridiques et sociales autour de la légalisation du mariage homosexuel. 
J'espère que ces articles sur la question de la représentation de la sexualité au Québec pourront susciter le débat et amorcer une réflexion constructive qui nous mènera au-delà de la panique morale sur un sujet toujours aussi passionnant, épineux et actuel. 